\title{
Intramolecular interactions between the AF3 domain and the C-terminus of the human progesterone receptor are mediated through two LXXLL motifs
}

\author{
X Dong ${ }^{1,2}$, J R G Challis ${ }^{2}$ and S J Lye L $^{1,2}$ \\ ${ }^{1}$ Program in Development and Fetal Health, Samuel Lunenfeld Research Institute, Mount Sinai Hospital, 600 University Avenue, Toronto, Ontario, \\ Canada M5G 1 X5 \\ ${ }^{2}$ Department of Physiology, University of Toronto, Ontario, Canada M5S 1A8 \\ (Requests for offprints should be addressed to S J Lye, Center for Women's and Infants' Health, Samuel Lunenfeld Research Institute, \\ Mount Sinai Hospital, 600 University Avenue, Toronto, Ontario, Canada M5G 1X5; Email:Lye@mshri.on.ca)
}

\begin{abstract}
The human progesterone receptor (PR) exists in two major forms, PRA and PRB, which differentially regulate gene transcription in a cell- and promoter-specific manner. The molecular mechanisms underlying this differential transcriptional activity have been attributed to the presence of a unique AF3 domain within PRB that may result in the two isoforms adopting different protein conformations. We demonstrate here that in myometrial cells, PRB exhibits strong progesterone-dependent transcriptional activity that is dependent on the presence of two LXXLL motifs within the AF3 domain. In vitro and in vivo protein interaction assays indicate that these motifs mediate the direct interaction between the AF3 domain and C-PR in a progesterone-dependent manner. Mutation of either of the LXXLL motifs or deletion of the last 30 amino acids within the C-terminus disrupts this interaction and progesterone-dependent transcriptional activity of PRB. Members of the p160 family of co-activators (such as GRIP-1) also interact with C-PR through their LXXLL motifs. However, GRIP-1 does not compete with $\mathrm{AF} 3$ but rather acts to synergize these two transactivation domains. Our data suggest that a failure to form an appropriate AF3-C-terminus interaction results in an inability of co-activators to induce maximal PR-dependent transactivation. The absence of an AF3 domain within PRA may account for its inability to activate progesterone-responsive genes, as well as its actions as a dominant trans-repressor.
\end{abstract}

Journal of Molecular Endocrinology (2004) 32, 843-857

\section{Introduction}

Progesterone is an essential regulator of the reproductive events associated with the establishment and maintenance of pregnancy. A withdrawal of progesterone's action must occur in order for labor to be initiated. In virtually all species this is achieved through a reduction in plasma progesterone levels at term, allowing increased expression of genes required for myometrial contractility and the onset of labor (Challis et al. 2000). Even though progesterone inhibits human myometrial contractility, there is no fall in plasma or tissue progesterone levels before labor. This paradox has led to the suggestion that there must be a 'functional withdrawal' of progesterone, possibly involving antagonism of the transcriptional activity of the progesterone receptor (PR) (Challis et al. 2000).

In humans, the biological response to progesterone is mediated by two major isoforms of the PR: PRA and PRB. These two isoforms are transcribed from distinct promoters within a single-copy PR gene: full-length PRB and a truncated PRA that lacks the first 164 amino acids of the N-terminus (Kastner et al. 1990). Studies in vivo involving mice carrying null mutations in the PR gene and transgenic mice overexpressing one of the two PR isoforms confirmed that the PRA and PRB isoforms serve different physiological functions (Conneely et al. 2001). Furthermore, wide fluctuations in the PRA:PRB ratio had been reported in female reproductive tissues during the normal 
menstrual cycle, and abnormal ratios are associated with highly malignant forms of reproductive tract cancer (Mangal et al. 1997).

In human myometrial cells, PRB is a strong transcriptional activator, while PRA is a weak activator (Pieber et al. 2001). Measurement of the expression levels of the two PR isoforms is complicated by the presence of multiple mRNA and protein species of the receptors. However, recent data suggest that there is an increase in the expression ratio of PRA to $\mathrm{PRB}$ in the human myometrium at term that blocks signaling through PRB, thereby inducing a functional withdrawal of progesterone (Mesiano et al. 2002). The molecular mechanism by which PRA and PRB possess different transactivation properties and by which an increase in PRA:PRB ratio induces a functional withdrawal remains to be determined.

Both forms of PR are identical in their centrally located DNA-binding domain (DBD), the C-terminal ligand-binding domain (LBD) and two independent transcriptional activation domains: a constitutive activation domain (AF1) in the N-terminus and a hormone-dependent AF2 in the LBD (McKenna et al. 1999). An inhibition function region (IF), located upstream of the AF1 domain, has also been characterized (Hovland et al. 1998). The IF domain directly inhibits the activation function of AF1 and AF2; it does not affect AF3, which is unique to PRB. In the absence of ligand, $\mathrm{PR}$ is maintained in an inactive state by its association with heat shock proteins (HSP) (McKenna et al. 1999). Binding of progesterone induces a conformational change, receptor dimerization, binding to DNA (at consensus sequences, progesterone response elements (PREs)) and transactivation of progesterone-responsive genes. PR regulates gene transcription through the direct interaction of the AF domains with the general transcription machinery, or indirectly by binding co-regulatory proteins. A large number of coactivators as well as co-repressors have been identified (McKenna \& O’Malley 2002). Coactivators bind to the LBDs through their consensus LXXLL (where L is a leucine and the X is any other amino acids) motifs (Heery et al. 1997). In most cell contexts, PRB is a stronger transcriptional activator, while PRA is weak activator and can functionally repress the transactivation of other steroid receptors (Vegeto et al. 1993). The functional differences between these two isoforms are attributed to the unique N-terminal transcriptional modulatory domain, AF3 (Sartorius et al. 1994). Recent data suggest that the N-terminus can interact in a hormonedependent manner with C-terminal of PR (Tetel et al. 1999). While co-activators do not mediate this interaction, they enhance $\mathrm{PR}$ transcriptional activity subsequent to the $\mathrm{N}-\mathrm{C}$ terminus association. Similar interactions between the N- and Ctermini of the estrogen receptor (ER) and androgen receptor (AR) have been reported (Benecke et al. 2000, He et al. 2000), which in the case of the AR is mediated by two motifs (FXXLF and WXXLF) within the N-terminus. Two similar signature LXXLL motifs are also found within the AF3 domain of PR, raising the question as to whether these motifs also mediate the intramolecular interaction within PRB.

In this study we show that the two LXXLL motifs within the AF3 domain are critical to the transcriptional activity of PRB in myometrial cells. These two motifs also mediate the intramolecular interaction between the AF3 domain and the C-PR as well as the co-activators mediating enhancement of progesterone-dependent transcriptional activity of PRB. The lack of an AF3 domain in PRA may alter these interactions and underlie its inability to effectively transactivate progesterone-dependent genes.

\section{Materials and methods}

\section{Plasmid constructions}

The expression vector pcDNA3 PRB was constructed from YephPRB vector (a gift from Dr Paul Walfish, Toronto, Canada). YephPRB was digested with $X h o \mathrm{I}$ and $E c o$ RI and ligated into the pSK vector. The recombined vector was then digested with $K p n \mathrm{I}$ and the fragment was inserted into the pcDNA3 vector (Invitrogen) at the same site. The pcDNA3 PRA vector was constructed by digesting YephPRB with BamHI. This fragment was inserted into the same site in pcDNA3. The PRE-Luc reporter vector was constructed as follows: the double-stranded oligonucleotide containing three consensus PRE sequences flanked by $B a m \mathrm{HI}$ and $B g l \mathrm{II}$ sites was linked into pBLCAT2 vector at the Bam HI site. The $3 \times$ PRE together with the TATA box sequence from pBLCAT2 was excised by SalI and $X h o I$, and ligated into pGL2 Basic (Promega) at 
the $X h_{0} \mathrm{I}$ site. The $\beta$-galactosidase reporter vectors pCH110 (Pharmacia) and CMV- $\beta$ Gal (Stratagene, BC, Canada) (CMV= cytomegalovirus) were used to control transfection efficiency. PCR amplification was performed with YephPRB as template to amplify AF3 (aa 1-164), C-PR (aa 556-933) and C-PR $\Delta 30$ (aa 556-903). The 5' sense primer contains an EcoRI site and an ATG start codon, while the $3^{\prime}$ sense primer contains the stop codon and SalI site. The PCR product of AF3 was ligated into pGEX5X-1 (Pharmacia) vector at the $E c o \mathrm{R} 1$ and Sall sites. C-PR and C-PR $\Delta 30$ were also cloned into pcDNA3 vector driven by $\mathrm{T} 7$ promoter. Site-directed mutagenesis was carried out by PCR amplification of the wild-type PR constructs with a Mutagenesis Kit (Stratagene). M1 defines the mutation LL58, 59AA. M2 defines the mutation LL1 18, 119AA. M1/2 defines the double mutation of both LXXLL motifs. The plasmid AF3-NLS was kindly provided by Dr Horwitz (Colorado, USA) as BUS-NLS, in which the AF3 domain is linked to a nuclear localization signal. Double mutations of both LXXLL motifs were made using BUS-NLS to construct the AF3 mNLS. All the recombinant plasmids and mutants were verified by DNA sequencing. The expression vector of GRIP-1 was a gift from Dr Walfish.

\section{Transient transfection and immunoblotting}

Syrian hamster myocyte (SHM) cells were maintained in DMEM plus 5\% fetal calf serum (Sigma) as described previously (Chen et al. 1995). For transient transfection, cells were seeded on 24-well plates in phenol red-free DMEM containing 5\% charcoal-treated fetal bovine serum (Hyclone, Logan, UT, USA). A total of $1 \mu \mathrm{g}$ DNA, containing $300 \mathrm{ng}$ reporter PRE-Luc and $200 \mathrm{ng}$ pCMV $\beta$-gal (Clontech), was introduced into each well using Exgen 500 (MBI Fermentas, ON, Canada). Four hours after transfection, medium was removed and cells were incubated with fresh DMEM plus $5 \%$ charcoal-treated fetal bovine serum and $10^{-8} \mathrm{M}$ progesterone for $30 \mathrm{~h}$. Cells were collected in $100 \mu \mathrm{l}$ lysis buffer (Promega), of which $10 \mu \mathrm{l}$ were used for luciferase and $\beta$-galactosidase activity assays. Luciferase activity was determined by using the luciferin reagent (Promega) according to the manufacture's protocol. Transfection efficiency was normalized to $\beta$-galactosidase activity. The expression levels of PR and its mutants derived from
GMV-driven expression vectors were tested by transient transfection into SHM cells, followed by immunoblotting with anti-PR monoclonal antibody AB-52 (Santa Cruz Biotechnology, Inc., Santa Cruz, CA, USA). Anti-Gal4 DBD antibody (Clontech) was used to detect the expression of Gal4 DBD-PR fusion proteins.

\section{In vitro protein interaction assay}

Glutathione S-transferase (GST) fusion proteins were expressed in $E$. coli strain BL21(DE3)pLysS according to the method described by the manufacturer (Pharmacia). Briefly, overnight cultures from single colonies were diluted 1:15 in $2 \times$ YT media containing $100 \mu \mathrm{g} / \mathrm{ml}$ ampicillin The cultures were grown at $37^{\circ} \mathrm{C}$ until an $\mathrm{OD}_{600}$ of 0.4 was reached, at which time isopropyl- $\beta$-Dthiogalactoside was added to a final concentration of $0.2 \mathrm{mM}$. After $3 \mathrm{~h}$ cultures were pelleted for $15 \mathrm{~min}$ at $10000 \mathrm{~g}$ at $4{ }^{\circ} \mathrm{C}$. Bacterial pellets were resuspended in $1 \mathrm{ml}$ NETN buffer $(0.5 \%$ NP-40, $1 \mathrm{mM}$ EDTA, $100 \mathrm{mM} \mathrm{NaCl}, 20 \mathrm{mM}$ Tris $\mathrm{pH}$ 8.0) plus a cocktail of protease inhibitors (Boehringer Mannheim) per $30 \mathrm{ml}$ culture and lysed by mild sonication. Sonicates were cleared by centrifugation for $10 \mathrm{~min}$ at $10000 \boldsymbol{g}$ and the supernatants were used for GST fusion protein purification and pull-down assays.

In protein pull-down assays, fusion proteins of GST AF3 and GST AF3 mutants were first immobilized on a glutathione-Sepharose 4B affinity matrix (Pharmacia). Then the matrix was incubated with rabbit reticulocyte lysate (Promega) containing C-PR (aa 556-933) and C-PR $\Delta 30$ (aa 556-903), transcribed and translated in the presence of $\left[{ }^{35} \mathrm{~S}\right]$ methionine at $4{ }^{\circ} \mathrm{C}$ overnight. In some reactions, $10^{-5} \mathrm{M}$ progesterone hormone was added. The matrix was then washed three times with cold NETN buffer before adding SDS loading buffer to elute associated proteins. The eluted proteins were separated on SDS-PAGE gel. Gels were treated with enhancer (NEN, Boston, MA, USA) dried and analyzed by autoradiography.

\section{Mammalian two-hybrid assay}

The PR AF3 domain (aa 1-164), its mutants AF3 m (containing a double mutation of LL58, 59AA and LL1 18, 119AA) and C-PR (aa 556-933) were amplified by PGR. The PGR products were 
then digested by EcoRI and ligated to $\mathrm{pM}$ and pVP16 (Clontech) at the same site. The constructs pM AF3/AF3 m, pM G-PR, VP AF3/AF3 m and VP C-PR were sequenced to confirm that the amplified PR fragments were inserted in frame. The G5-Luc reporter vector contained a TATA box and five copies of the Gal4 DNA binding site (a kind gift from Dr Govindan, University of Laval, Quebec City, Canada).

In the two-hybrid assay, SHM cells were cultured in DMEM plus 10\% charcoal-stripped serum and seeded in 24-well plates. DNAs (300 ng G5-Luc, $200 \mathrm{ng}$ pCH110, $300 \mathrm{ng}$ VP and/or pM fusion constructs) were introduced into cells by Exgen 500. Four hours after transfection, medium was removed and cells were incubated with fresh DMEM medium with or without $10^{-7} \mathrm{M}$ progesterone for $30 \mathrm{~h}$. The basal luciferase expression in the absence of $\mathrm{pM}$ and VP16 constructs was normalized to the value of $1 \cdot 0$. Specific luciferase expression resulting from $\mathrm{AF} 3$ and $\mathrm{C}-\mathrm{PR}$ fusion protein interactions was calculated as the fold induction over the basal luciferase activity.

\section{Statistics}

Data are presented as means \pm S.D. from three independent experiments. Significance was determined by either one- or two-way ANOVA using the software SIGMASTAT.

\section{Results}

\section{Differential transcriptional activities of PRB and PRA isoforms in myometrial cells}

SHM cells were co-transfected with PRA or PRB expression vectors and the progesterone-responsive reporter vector PRE-Luc (Fig. 1A). Cells were treated with either vehicle or $10^{-8} \mathrm{M}$ progesterone for at least $30 \mathrm{~h}$. Progesterone treatment of cells co-transfected with PRB caused a dramatic increase (17-fold compared with vehicle) in reporter gene expression, which was proportional to the mass of the input plasmid. In contrast, progesterone induced a relatively minor activation (2- to 4-fold) of reporter expression in the presence of PRA. Co-transfection of PR isoforms into SHM cells demonstrated that PRA could antagonize the transactivational property of PRB (Fig. 1B). Progesterone-dependent activation by PRB was decreased approximately $10 \%$ when the ratio of input plasmid of PRB:PRA was 10:1. Increasing the input of PRA plasmid to 5:1 (PRB:PRA) resulted in a reduction in luciferase activity by $50 \%$ and at a PRB:PRA ratio of 5:2 luciferase activity was reduced to $20 \%$ of the PRB level alone. Western blot analysis demonstrated the appropriate expression of PRA and PRB proteins in these transient transfection studies, indicating that the changes in luciferase activity were not due to a failure in expression of the PR isoforms (Fig. 1C).

\section{Transcriptional activity of PR isoforms depends on two LXXLL motifs in the AF3 domain}

Two LXXLL motifs are located at aa 55-59 and 115-119 within the AF3 domain. These signature motifs are usually found in co-activators and are required for their binding to the LBD. This raised the possibility that these two LXXLL motifs might mediate the interaction between AF3 and C-PR. The importance of these motifs to PR transcriptional activity was investigated in transient transfection assays using $3 \times$ PRE-Luc as a reporter gene. Mutation of either or both of these motifs (LL58, 59AA and LL1 18, 119AA) resulted in a significant decrease in luciferase expression (Fig. 2A) to around $20 \%$ of the wild-type PRB, a level similar to that of the wild-type of PRA (around 15\% of PRB). The differences in reporter expression were not due to differences in input plasmid expression, since similar levels of PR proteins were observed by Western blotting using the AB-52 antibody (Fig. 2B). To further investigate whether these two LXXLL motifs would impact the function of the AF3 domain, we used a one-hybrid assay system, in which the wild-type AF3 domain was fused to the Gal 4 DBD (pM AF3). G5-Luc was used as a reporter vector. We observed that $\mathrm{pM} A \mathrm{AF} 3$ led to a $>30$-fold induction of G5-Luc reporter expression compared with the empty $\mathrm{pM}$ vector and about $60 \%$ of the positive control vector (pM3 VP16). This transactivation function of AF3 domain also required intact LXXLL motifs, since mutation of either or both of these motifs (pM AF3 M1, pM AF3 M2 and pM AF3 M1/2) greatly impaired (up to $60 \%$ deduction) reporter expression (Fig. 3A). The protein expression levels derived from the $\mathrm{pM}$ AF3 and its mutants were tested by transient transfections of these constructs into SHM cells. Western blot analysis of protein lysates $(50 \mu \mathrm{g})$ from these transfections revealed similar levels of 


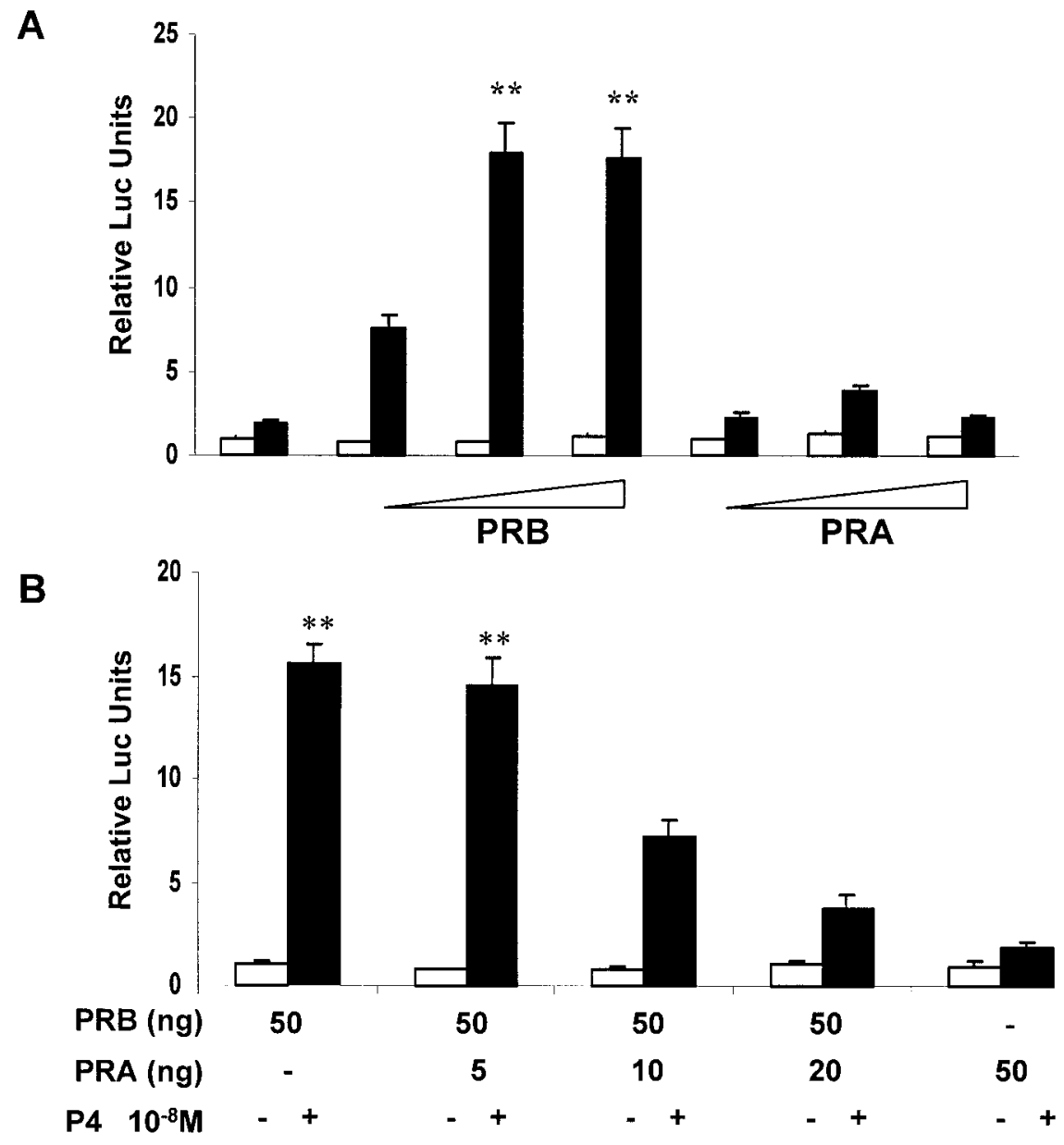

C

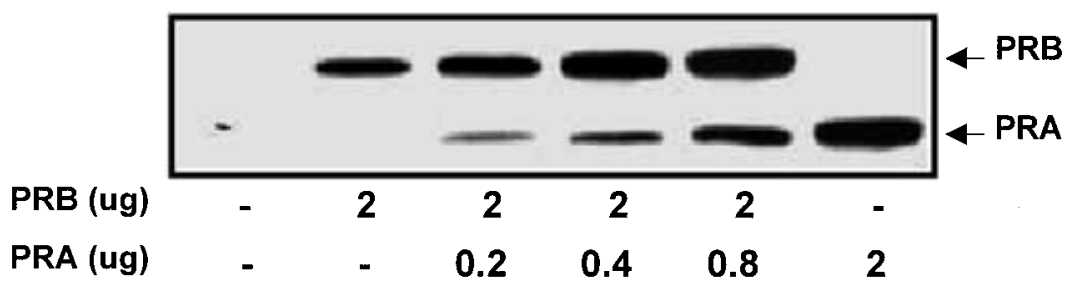

Figure 1 Differential transcriptional activities of PRB and PRA isoforms in myometrial cells. SHM cells were transfected with increasing amounts of PRB or PRA expression vector $(10,20,50 \mathrm{ng})$ (A) or SHM cells were transfected with $50 \mathrm{ng}$ expression vector of PRB with increasing amounts $(5,10,20$ and $50 \mathrm{ng})$ of PRA (B).

Expression vectors were co-transfected with $300 \mathrm{ng} 3 \times \mathrm{PRE}$-Luc luciferase report vector and $200 \mathrm{ng}$ CMV- $\beta$ Gal. Four hours after transfection, the medium was replenished and cells were either untreated (open columns) or treated (filled columns) with $10^{-8} \mathrm{M}$ progesterone (P4) for $30 \mathrm{~h}$. Luciferase activities were normalized to $\beta$-galactosidase activities and were plotted as the fold induction over the promoter basal activity in the absence of PR expression vector and progesterone. Values are shown as means \pm S.D. from three independent experiments. Statistical analysis was performed using two-way ANOVA and a Student-Newman-Keuls test; ${ }^{\star \star}{ }^{*}<0.001, n=3$. (C) The expression of PRA and PRB derived from expression vectors with indicated amounts of plasmids were tested by transient transfection into SHM cells; the same amount of protein lysates $(60 \mu \mathrm{g})$ was separated on $8 \%$ SDS gel. After transfer, membrane was blotted with anti-PR monoclonal antibody AB-52. 
A

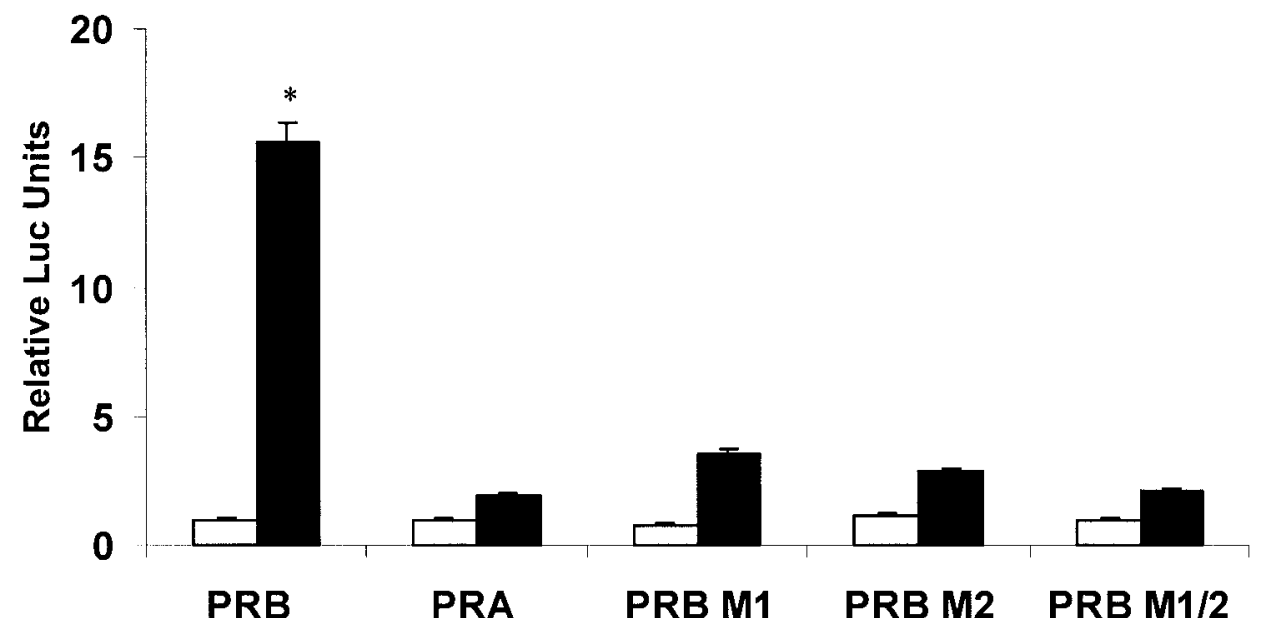

B

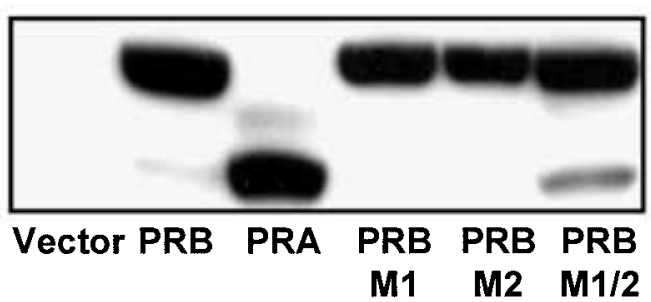

Figure 2 Transcriptional activity of PR isoforms depends on two LXXLL motifs in the AF3 domain. (A) SHM cells were transfected with $50 \mathrm{ng}$ of expression vectors of PRB, PRA and PRB mutants. Expression vectors are co-transfected with $300 \mathrm{ng} 3 \times \mathrm{PRE}-\mathrm{LuC}$ luciferase report vector and $200 \mathrm{ng}$ CMV- $\beta$ Gal. Four hours after transfection, the medium was replenished and cells were either untreated (open columns) or treated (filled columns) with $10^{-8} \mathrm{M}$ progesterone for $30 \mathrm{~h}$. Luciferase activities were normalized to $\beta$-galactosidase activities and were plotted as the fold induction over the promoter basal activity in the absence of PR expression vector and progesterone. Values are shown as means \pm S.D. from three independent experiments. Statistical analysis was performed using two-way ANOVA and a Student-Newman-Keuls test; ${ }^{*} P<0 \cdot 05, n=3$. Note: The LXXLL motifs were mutated individually (M1 or $\mathrm{M} 2$ ) or in combination (M1/2) to produce of double mutant. (B) The expression levels of PR and its mutant-derived expression vectors were tested by transient transfection in SHM cells. The same amount $(60 \mu \mathrm{g})$ of whole cell lysates was separated on $8 \%$ SDS gel, followed by immunoblotting with anti-PR monoclonal antibody AB-52.

expressed proteins (pM AF3, pM AF3 M1, M2 and M1/2) when blotted with anti-Gal4 DBD antibody (Fig. 3B).

\section{Two LXXLL motifs mediate the AF3 domain and $\mathrm{C}$-terminus interaction of PRB in vitro}

Horwitz (Tung et al. 2001) proposed several possible mechanisms by which mutation of the
LXXLL motifs within AF3 could disrupt the transactivation function of PRB, including the possibility of a direct interaction of the $\mathrm{N}$ - and C-termini mediated by the LXXLL motifs. To test this possibility we used a GST AF3 fusion protein as bait to pull down C-PR. A significant progesterone-dependent interaction of C-PR with AF3 was detected (Fig. 4A). This interaction was disrupted by deletion of the most C-terminal 30 

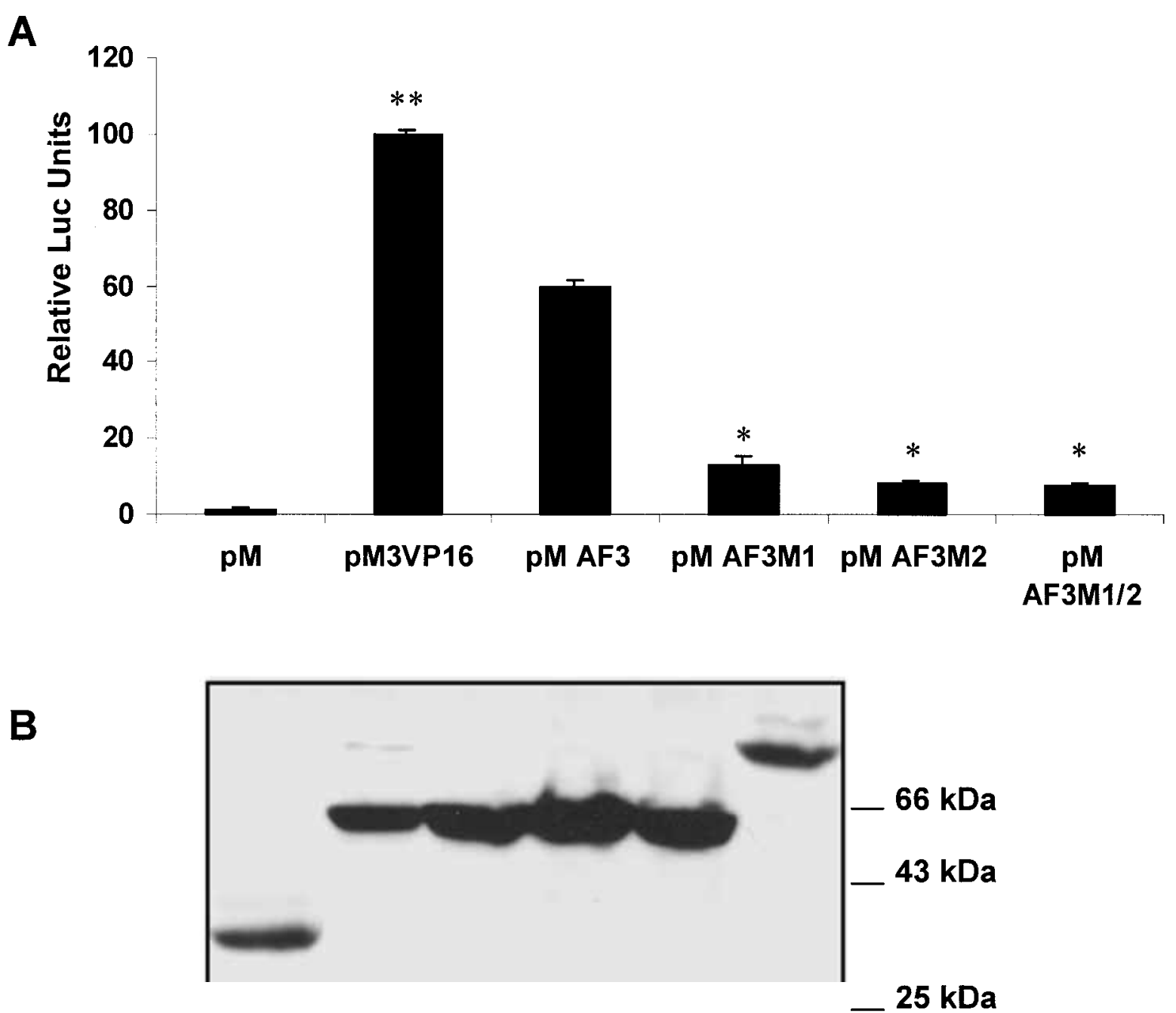

\section{pM $\quad$ pM $\quad$ pM $\quad$ pM $\quad$ pM $\quad$ pM \\ AF3 AF3M1 AF3M2 AF3M1/2 C-PR}

Figure 3 The LXXLL motifs are critical for the transactivation of the AF3 domain. (A) The pM AF3/AF3 mutants were co-transfected with G5-Luc reporter. Luciferase activities were normalized to $\beta$-galactosidase activities and were plotted as the fold induction over the empty pM vector. The pM3 VP16 was used as positive control and plotted as $100 \%$. Values are shown as means \pm S.D. from three independent experiments. Statistical analysis was performed by one-way ANOVA and a Student-Newman-Keuls test; ${ }^{* \star} P<0.001$, ${ }^{*} P<0.05, n=3$. Note: The LXXLL motifs were mutated individually (M1 or M2) or in combination (M1/2) to produce a double mutant. (B) pM AF3/AF3 mutants and pM C-PR were transiently transfected into SHM cells. The proteins derived from these vectors were tested by immunoblotting with mouse monoclonal anti-Gal4 DBD antibody.

amino acids (aa 903-933) of C-PR. The association of the AF3 domain with C-PR was also dependent upon the integrity of the LXXLL motifs within AF3, since mutation of either of these elements decreased the association by up to $50 \%$, while mutation of both dramatically decreased the association by up to $80 \%$ compared with the wild-type AF3 (Fig. 4B). These differences were not due to different inputs of GST fusion proteins since electrophoresis of the same mass of GST fusion 
A

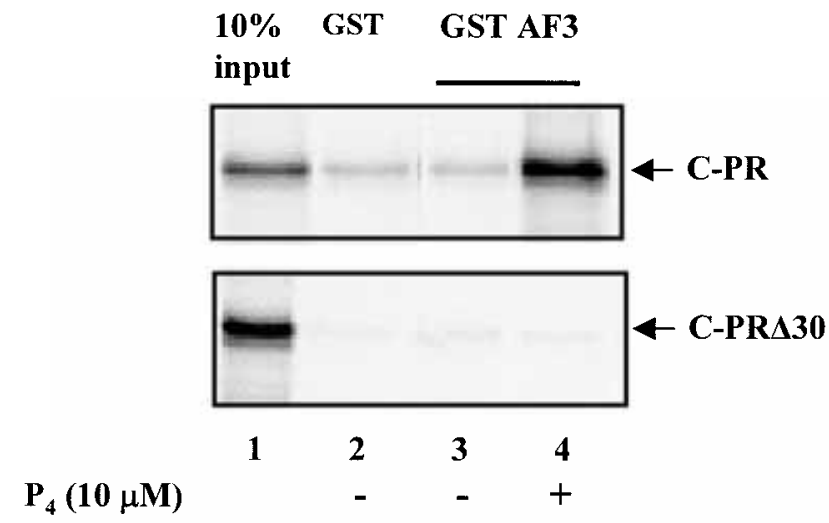

B

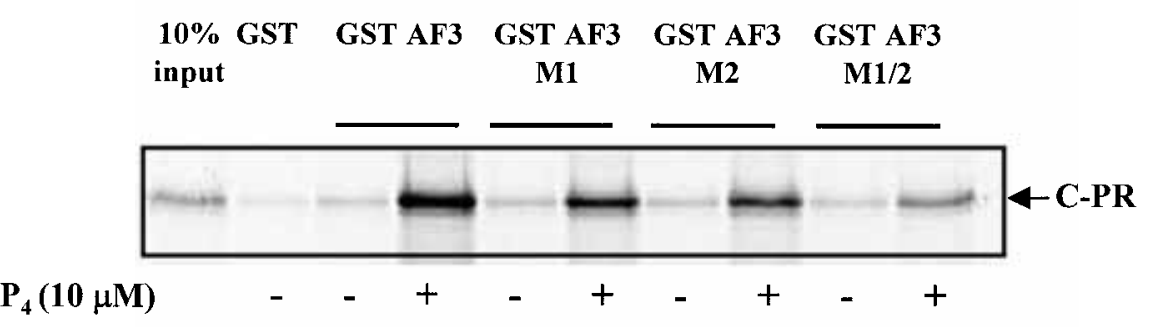

C

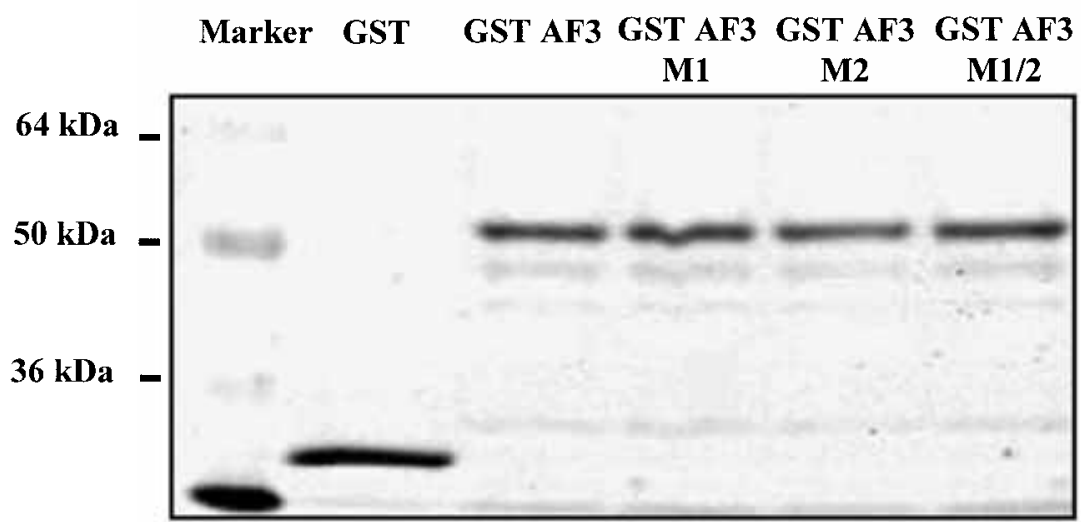

Figure $4 \mathrm{AF} 3$ domain and $\mathrm{C}$-terminus of PR interact directly through two LXXLL motifs. (A) GST or GST AF3 fusion proteins were immobilized on glutathioneSepharose 4B beads and incubated with $10 \mu \mathrm{l}{ }^{35}$ S]methionine-labeled C-PR or C-PR $\triangle 30$ protein. (B) GST AF3 and its mutants were immobilized on glutathione-Sepharose $4 \mathrm{~B}$ beads and incubated with $10 \mu \mathrm{l}\left[{ }^{35} \mathrm{~S}\right]$ methionine-labeled C-PR. Progesterone hormone was added to a final concentration of $10^{-5} \mathrm{M}$ as indicated. After overnight incubation at $4{ }^{\circ} \mathrm{C}$, the matrix was washed with cold NETN buffer three times. The associated protein were eluted by adding SDS loading buffer and separated on $12 \%$ acrylamide gel and analyzed by autoradiography. Note: The LXXLL motifs were mutated individually (M1 or M2) or in combination (M1/2) to produce a double mutant. (C) Coomassie staining of the same amount of purified GST fusion protein used in a GST pull-down assay on SDS-PAGE. 
proteins produced similar densities of protein bands (Fig. 4C). Thus, these data confirm that the AF3 domain (through its two LXXLL motifs) can directly interact with C-PR.

\section{The AF3 and C-terminus of PR functionally interact in vivo}

A mammalian two-hybrid assay was also conducted to determine whether the AF3 and C-terminus of PRB could functionally interact within SHM cells. The pM AF3 construct increased reporter gene expression by approximately 30-fold compared with empty $\mathrm{pM}$ vector. Addition of C-PR fusion protein without progesterone did not result in significant induction of luciferase activity compared with pM AF3 (Fig. 5A). However, co-transfection of VP C-PR in the presence of progesterone caused a dramatic increase (90-fold) of luciferase activity. These results indicate that AF3 interacts with $\mathrm{C}-\mathrm{PR}$ in vivo in a progesterone-dependent manner. Double mutations of the two LXXLL motifs (AF3 $\mathrm{m}$ ) reduced the transactivation capability of the AF3 domain and disrupted the AF3-C-PR interaction, even in the presence of progesterone. Similar findings were obtained when the C-terminal segment was fused to the $\mathrm{pM}$ vector and co-transfected with VP AF3 (Fig. 5B). The pM $\mathrm{C}$-PR vector alone induced a $\sim 20$-fold increase in luciferase activity, a level that was not significantly increased by progesterone. However, addition of VP AF3 caused a dramatic induction of 80-fold in the presence (but not absence) of progesterone, confirming the interaction between AF3 and C-PR. Consistently, VP AF3 m did not interact with G-PR. These studies support the findings of the in vitro protein-protein interaction assay. Thus the LXXLL motifs are essential for the transactivation function of the AF3 domain and its functional interaction with the $\mathrm{C}$-terminus of PRB.

\section{Co-activators enhance AF3 and C-terminus functional interaction}

Our data show that the AF3 domain interacts with C-PR through its LXXLL motifs. Since coactivators also use this motif to interact with the LBD of steroid receptors, we questioned whether co-activators might interfere with the interaction between the AF3 domain and C-PR. In order to clarify this possibility, we analyzed the effects of co-expression of GRIP-1 on AF3-C-PR interactions. The pM C-PR and VP AF3 or VP AF3 m were co-transfected with GRIP-1 expression vector in SHM cells. Competition by GRIP-1 for the AF3-mediated interaction with $\mathrm{C}$-PR would reduce the stimulation of G5-Luc expression by $\mathrm{pM}$ C-PR plus VP AF3. However, we observed that GRIP-1 enhanced, rather than inhibited, luciferase expression induced by the interaction of AF3 and C-PR (Fig. 6A), suggesting that GRIP-1 does not compete with AF3 for the LXXLL binding site in C-PR. Mutation of the LXXLL motifs in AF3 rendered this domain ineffective in enhancing the interaction between GRIP-1 and C-PR, demonstrating the requirement for these motifs for the efficiency of GRIP-1 co-activation of C-PR. To confirm this effect a similar two-hybrid assay was also performed, in which the AF3 domain or its mutant was tethered to Gal4 DBD (Fig. 6B) and subsequently co-transfected with VP C-PR in the presence or absence of GRIP-1. As expected we observed that VP C-PR could form a complex with AF3 but not with its mutant AF3 m. GRIP-1 enhanced the interaction between AF3 and C-PR, but since AF3 $\mathrm{m}$ was incapable of interacting with C-PR, GRIP-1 failed to induce expression of G5-Luc.

Since VP16 and GRIP-1 both contain activation domains it is not informative to use a mammalian two-hybrid assay to assess the impact of AF3 on GRIP-1/C-PR interaction. We therefore chose a one-hybrid assay to investigate the potential modulatory role of AF3. Vectors (pM PRB and pM PRA) containing PRB and PRA cDNA downstream from the Gal4 DBD were constructed and their expression confirmed by Western blotting following transient transfection in SHM cells (Fig. 7A). GRIP-1 significantly enhanced pM PRB-induced transactivation of G5-Luc. In contrast, GRIP-1 had little effect on the limited transactivational activity of pM PRA.

To further explore the significance of the AF3 domain on the interaction and co-activation of the C-PR by GRIP-1, we investigated the impact of overexpression of AF3 on the interaction between GRIP-1 and C-PR. A construct containing the AF3 domain linked to a nuclear localization signal (AF3-NLS) was obtained from Dr K Horwitz and both of the LXXLL motifs in AF3 were mutated using site-directed mutagenesis. SHM cells were transfected with expression vectors for $\mathrm{pM} \mathrm{C}$-PR in 
A

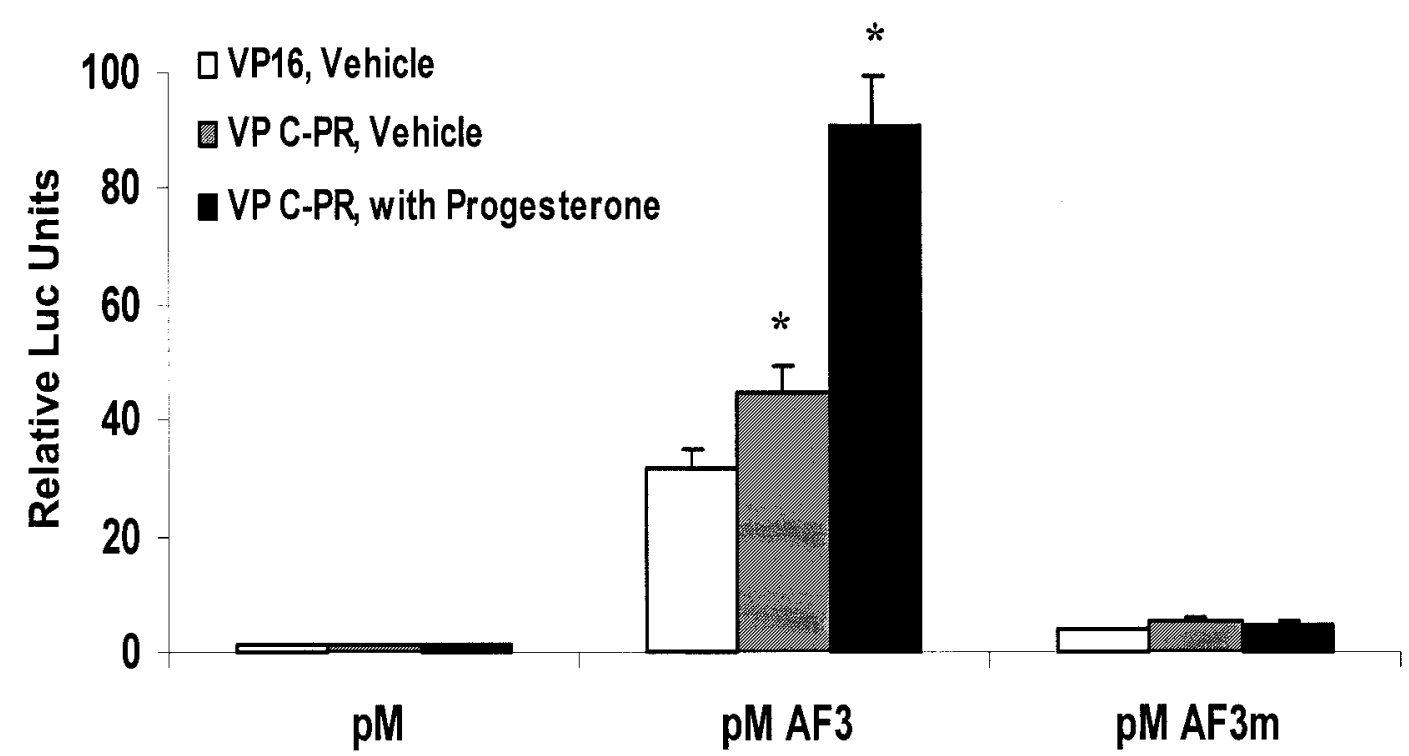

B

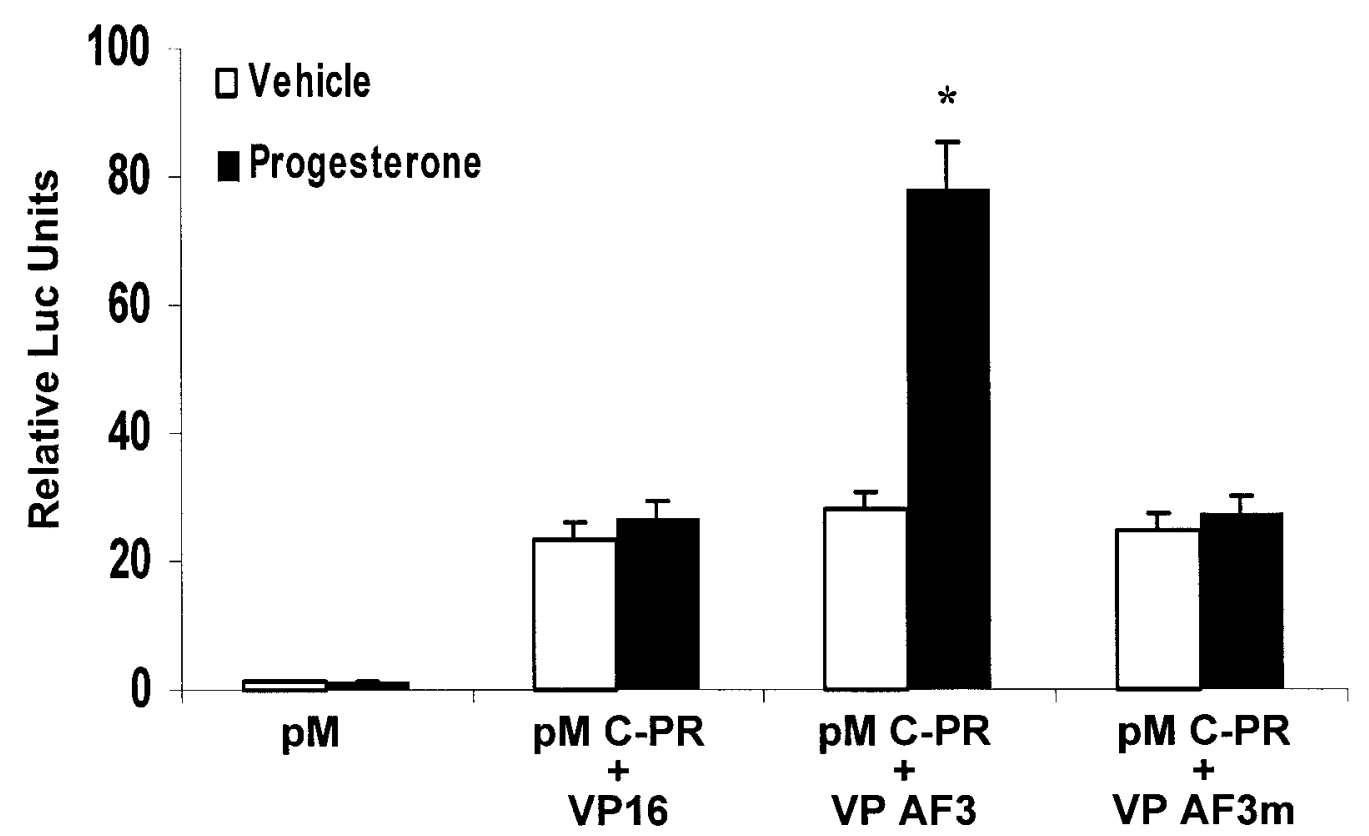

Figure 5 Interaction of the AF3 domain and C-terminus of PR detected by mammalian two-hybrid assay. (A) pM AF3 or pM AF3 m (containing mutations of both LXXLL motifs) was co-transfected with VP16 or VP C-PR (PR 556-933 ) or (B) pM C-PR ( PR $\left._{556-933}\right)$ was co-transfected with VP16 or VP AF3/AF3 m. They were transiently transfected with G5 Luc and pCH110 report vector in SHM cells. Four hours after transfection, cells were treated with or without $10^{-7} \mathrm{M}$ progesterone for at least $30 \mathrm{~h}$. Luciferase activities were normalized to $\beta$-galactosidase activities and were plotted as the fold induction over the empty pM vector. Values are shown as means \pm S.D. from three independent experiments. Statistical analysis was performed using two-way ANOVA and a Student-Newman-Keuls Test; ${ }^{*} P<0.001, n=3$. 
A

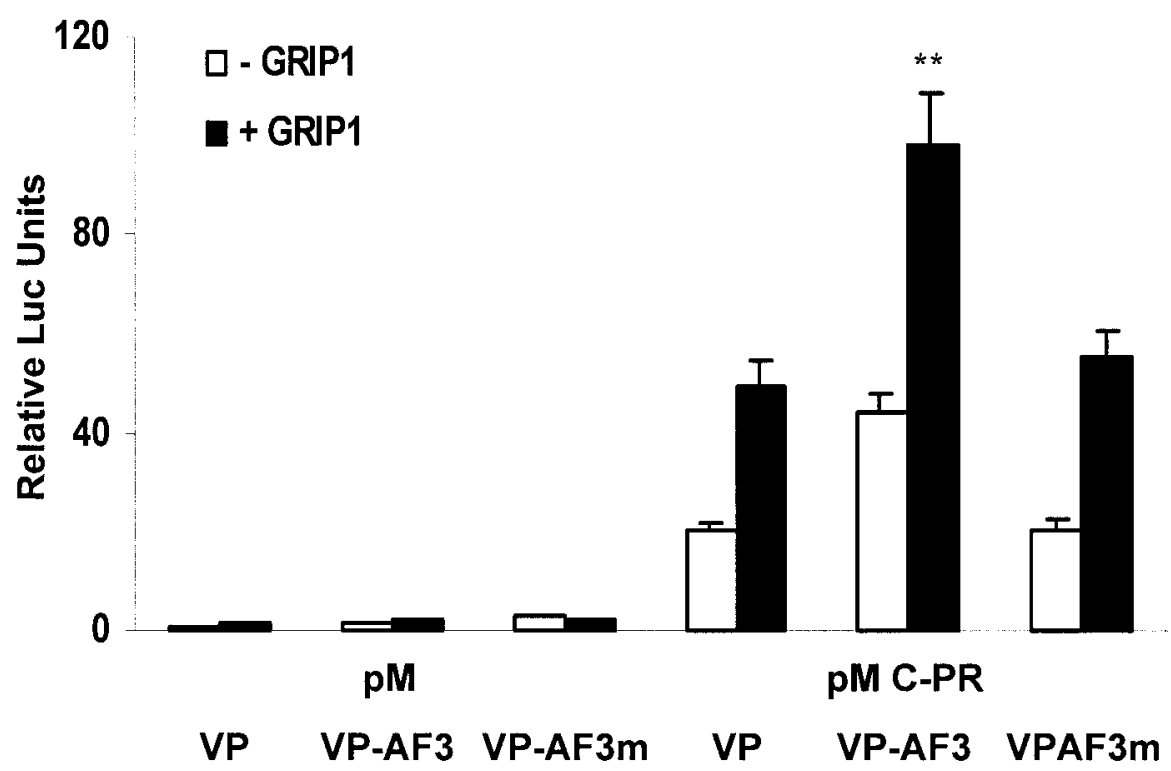

B

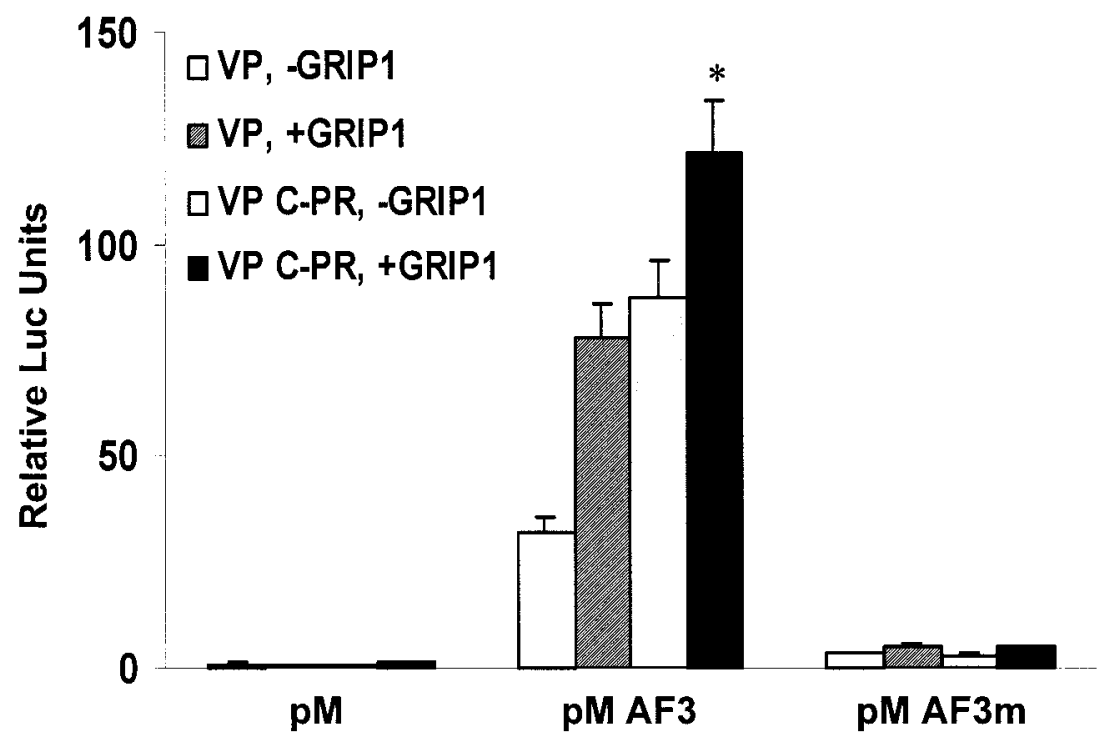

Figure 6 GRIP-1 enhances the functional interaction between the AF3 domain and C-PR. (A) SHM cells were transiently transfected with pM AF3 or its mutant pM AF3 m (containing mutations of both LXXLL motifs) with VP C-PR, or (B) pM C-PR with VP AF3 or VP AF3 m. Cells were also co-transfected with or without GRIP-1 expression vector together with G5 Luc and $\mathrm{pCH} 110$ report vectors. Cells were then treated with $10^{-7} \mathrm{M}$ progesterone for at least $30 \mathrm{~h}$. Luciferase activity was normalized to $\beta$-galactosidase activity and data are represented as fold induction over the luciferase reading in the transfection of empty $\mathrm{pM}$. Values are shown as means \pm S.D. from three independent experiments. Statistical analysis was performed using one- or two-way ANOVA and a Student-Newman-Keuls test;

${ }^{*} P<0.001,{ }^{*}<0.05, n=3$. 
A

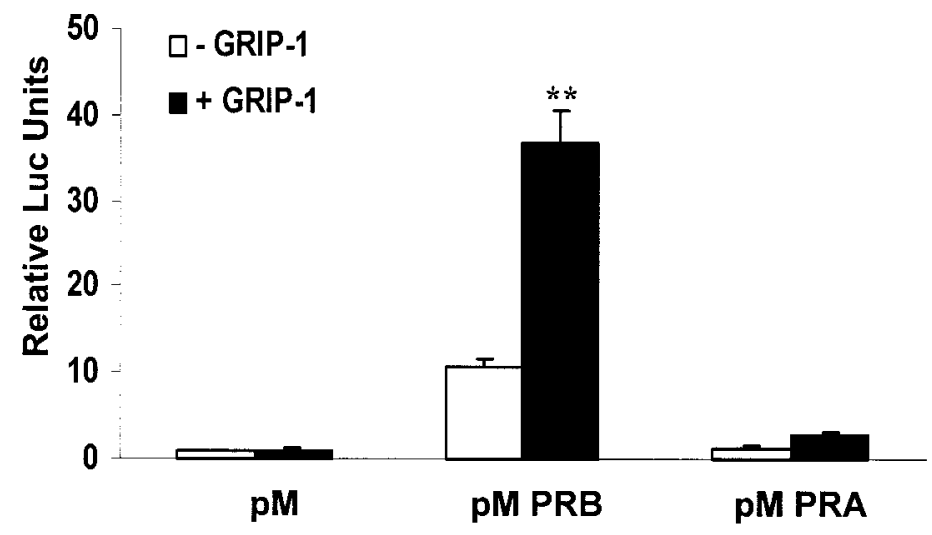

B

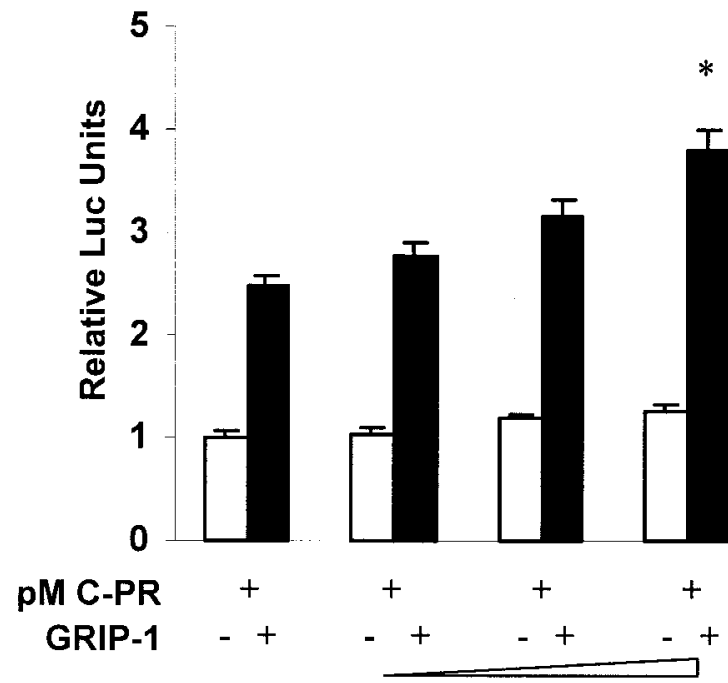

AF3-NLS

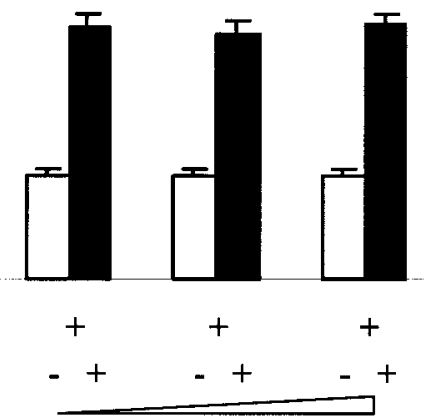

AF3m-NLS

Figure 7 The AF3 domain enhances GRIP-1 interacting and co-activating C-PR. (A) SHM cells were transiently transfected with pM PRA or pM PRB in the presence or absence of GRIP-1 expression vector. The expressed proteins derived from PM PRA and pM PRB expression vectors were tested by immunoblotting with mouse monoclonal anti-PR antibody AB-52 (A, lower part). (B) SHM cells were transiently transfected with pM C-PR and increasing amounts $(0.1,0.2,0.5 \mu \mathrm{g})$ of expression vector of AF3-NLS or AF3 m-NLS (containing mutations of both LXXLL motifs) in the presence or absence of GRIP-1. Cells were then treated with $10^{-7} \mathrm{M}$ progesterone for at least $30 \mathrm{~h}$. Luciferase activity was normalized to $\beta$-galactosidase activity and data are represented as fold induction over the luciferase reading in the transfection of empty vector pM in (A) or pM C-PR in (B). Values are shown as means \pm S.D. from three independent experiments. Statistical analysis was performed using one-way ANOVA and a Student-Newman-Keuls test; ${ }^{* *} P<0.001,{ }^{*}<0.05$, $n=3$. 
the presence or absence of GRIP-1 together with increasing plasmid mass $(0 \cdot 1,0.2$ and $0.5 \mu \mathrm{g})$ of AF3-NLS or AF3 m-NLS. In the absence of GRIP-1, overexpression of AF3-NLS resulted in minimal enhancement of C-PR-induced transactivation. However, in the presence of GRIP-1, AF3-NLS (but not AF3 m-NLS) significantly increased the transactivation of the G5-Luc reporter by C-PR. The increment in luciferase expression was proportional to the level of AF3-NLS input plasmid. These data suggest that GRIP-1 and AF3 can each enhance the other's interaction with C-PR and thereby the transactivational function of $\mathrm{PR}$.

\section{Discussion}

Accumulating evidence suggests that full activation of steroid receptors involves activation domains in both the $\mathrm{N}$ - and $\mathrm{C}$-terminus. Interactions between the N-terminus of PRB and C-PR have been shown to be more efficient than those between PRA and C-PR (Tetel et al. 1999), implying additional binding sites exist within the unique AF3 domain within the N-terminus of PRB. The presence of two LXXLL motifs within the AF3 domain led us to question whether these two motifs might mediate the intramolecular interaction within PRB. Using both in vitro GST pull-down and in vivo two-hybrid assays our data demonstrate that these two LXXLL motifs do indeed mediate AF3-C-PR interaction and that this interaction is dependent upon progesterone. Moreover, our data indicate that these LXXLL motifs are not only required for AF3-C-PR interaction but also mediate the transactivational function of PRB, since mutation of either or both of these motifs impairs function of this receptor. The p160 family of steroid receptor co-activators such as GRIP-1 have been identified as proteins that interact with the LBD through multiple LXXLL motifs (Hong et al. 1999), each of which can bind the LBD independently. GRIP-1 can also act as an adapter to modulate the interaction between the $\mathrm{N}$ - and $\mathrm{C}$-terminus of PR. We further investigated whether these co-activators might compete with AF3 to bind C-PR, since both GRIP-1 and AF3 contain LXXLL motifs. Interestingly, rather than compete, our data indicate that GRIP-1 enhances the functional interaction between the AF3 and C-PR.
Moreover, overexpression of the AF3 domain also enhances GRIP-1 co-activation of G-PR, an effect that is dependent upon the two LXXLL motifs in AF3. Therefore, although both AF3 and GRIP-1 possess the same LXXLL motifs, they do not interact with C-PR in the same way, or at least not through the same binding site. Thus, our data support the conclusion that the interaction between the N-terminus and $\mathrm{C}$-terminus of PRB is mediated directly through the LXXLL motifs in the AF3 domain or indirectly through association with co-activators, such as GRIP-1. Optimal transactivation by $\mathrm{PR}$ requires interaction between all three components, namely the AF3 and C-PR domains of $\mathrm{PR}$ and the co-activators.

The Horwitz laboratory has identified an IF within PR that is able to inhibit the activation function of the AF1 and AF2 domains (Hovland et al. 1998). However, the IF domain does not block the activation function of AF3 and it has been suggested that this difference might be one mechanism by which PRA and PRB isoforms exhibit different transcriptional activities. Our data would support the possibility that the intramolecular interaction between AF3 and C-PR might mask (or prevent the inhibitory function of the IF domain and thus provide increased access of PRB to co-activators. O'Malley and colleagues (Vegeto et al. 1992) have suggested that the extreme C-terminus of PR exhibits a repressive function on PR transcriptional activity, possibly through altered conformation of the LBD, which in turn promotes recruitment of co-repressors. Our protein pulldown assays showed that the association between AF3 and G-PR requires the most-terminal 30 amino acids of PR, raising the possibility that, through interaction with these amino acids, AF3 might also modulate the function of the LBD. We speculate that upon binding to G-PR, through the LXXLL motifs, the AF3 domain could influence the conformation of both the LBD and the AF3 domain itself so as to form a more stable complex with transcriptional co-activators. We further speculate that the association of progesterone with the LBD is permissive to the interaction between the AF3 domain and LBD and that a subsequent change in receptor conformation could act to repel co-repressors and de-repress their transactivational properties.

Interaction between $\mathrm{N}$ - and C-termini of steroid receptors represents a general mechanism 
mediating their activation as transcription factors. In the case of the AR and PR these domains interact directly in the presence of ligand (He et al. 1999, Tetel et al. 1999). In AR, two motifs, FQNLF and WHTLF (analogous to the LXXLL motifs of PR), mediate this interaction (He et al. 2000). There is also substantial evidence from multiple steroid receptors for indirect interaction between $\mathrm{N}$ - and C- termini mediated by co-activators, especially the p160 family (Berrevoets et al. 1998, Onate et al. 1998, Bevan et al. 1999, Benecke et al. 2000, Oberste-Berghaus et al. 2000). Interaction between co-activators and steroid receptors involves multiple sites within the co-activators, including, but not limited to, the LXXLL motifs. Co-activators have been shown to activate the N-terminal domain of ER, PR, thyroid receptor and AR in a ligand-independent manner and also interact with and activate the AF2 domain in a ligand-dependent manner. Presumably, these interactions result in more stable conformation and therefore act cooperatively to achieve maximum transcriptional activity.

To achieve a successful parturition, the action of progesterone signal has to be blocked to initiate labor. In humans, withdrawal of progesterone signaling is likely to be controlled at the level of its receptors. Our data show that strong transcriptional activity of PRB in myometrial cells is due to the two LXXLL motifs within its AF3 domain. These motifs also enable intramolecular interaction between the $\mathrm{N}$ - and $\mathrm{C}$-terminus of PRB, possibly leading to a conformational change of the LBD in the presence of progesterone. We speculate that the absence of an AF3 domain in PRA might account for its weak transactivational property in myometrial cells. As such, PRA might antagonize PRB-mediated signal transduction through formation of inactive heterodimers or by competing with PRB for binding to PREs. Recently published data demonstrate that there is an increased ratio of PRA to PRB expression at the human term labor, implying the ratio of PRA:PRB may an important factor for withdrawal of progesterone (Mesiano et al. 2002). Enhancement of N- and C-terminus activation domains of $\mathrm{PR}$ by co-activators represents another critical factor controlling progesterone signaling. Recent data show significant decrease in the expressions of PR co-activators such as GRIP-1 and SRC-3 in the fundal myometrium of women in labor (Condon et al. 2003). Our current data, together with that of other investigators, suggest mechanisms by which an increase in the ratio of myometrial PRA to $\mathrm{PRB}$ and/or a decrease in expression of PR co-activators in the uterus might induce a functional withdrawal of progesterone signaling and an increased sensitivity of the myometrium to contractile stimuli.

\section{Acknowledgement}

This work was supported by a grant from the Canadian Institute of Health Research (MOP-42378).

\section{References}

Benecke A, Chambon P \& Gronemeyer H 2000 Synergy between estrogen receptor alpha activation functions $\mathrm{AF} 1$ and $\mathrm{AF} 2$ mediated by transcription intermediary factor TIF2. EMBO fournal 2 151-157.

Berrevoets CA, Doesburg P, Steketee K, Trapman J \& Brinkmann AO 1998 Functional interactions of the AF2 activation domain core region of the human androgen receptor with the aminoterminal domain and with the transcriptional co-activator TIF2 (transcriptional intermediary factor 2). Molecular Endocrinology 12 $1172-1183$.

Bevan CL, Hoare S, Claessens F, Heery DM \& Parker MG 1999 The AF1 and AF2 domains of the androgen receptor interact with distinct regions of SRC1. Molecular and Cellular Biology 19 8383-8392.

Challis JRG, Matthews SG, Gibb W \& Lye SJ 2000 Endocrine and paracrine regulation of birth at term and preterm. Endocrine Reviews 21 514-550.

Chen ZQ, Lefebvre D, Bai XH, Reaume A, Rossant J \& Lye SJ 1995 Identification of two regulatory elements within the promoter region of the mouse connexin 43 gene. Fournal of Biological Chemistry 270 3863-3868.

Condon JC, Jeyasuria P, Faust JM, Wilson JW \& Mendelson CR 2003 A decline in the levels of progesterone receptor co-activators in the pregnant uterus at term may antagonize progesterone receptor function and contribute to the initiation of parturition. PNAS 100 9518-9523.

Conneely OM, Mulac-Jericevic B, Lydon JP \& De Mayo FJ 2001 Reproductive functions of the progesterone receptor isoforms: lessons from knock-out mice. Molecular and Cellular Endocrinology 179 97-103.

He B, Kemppainen JA, Voegel JJ, Gronemeyer H \& Wilson EM 1999 Activation function 2 in the human androgen receptor ligand binding domain mediates interdomain communication with the $\mathrm{NH}(2)$-terminal domain. Fournal of Biological Chemistry 274 37219-37225.

He B, Kemppainen JA \& Wilson EM 2000 FXXLF and WXXLF sequences mediate the NH2-terminal interaction with the ligand binding domain of the androgen receptor. Fournal of Biological Chemistry 275 22986-22994.

Heery DM, Kalkhoven E, Hoare S \& Parker MG 1997 A signature motif in transcriptional coactivators mediates binding to nuclear receptors. Nature $\mathbf{3 8 7} 733-736$

Hong H, Darimont BD, Ma H, Yang L, Yamamoto KR \& Stallcup MR 1999 An additional region of coactivator GRIP-1 required 
for interaction with the hormone-binding domains of a subset of nuclear receptors. Fournal of Biological Chemistry 274 3496-3502.

Hovland AR, Powell RL, Takimoto GS, Tung L \& Horwitz KB 1998 An N-terminal inhibitory function, IF, suppresses transcription by the A-isoform but not the B-isoform of human progesterone receptors. Fournal of Biological Chemistry $\mathbf{2 7 3}$ 5455-5460.

Kastner P, Krust A, Turcotte B, Stropp U, Tora L, Gronemeyer H \& Chambon P 1990 Two distinct estrogen-regulated promoters generate transcripts encoding the two functionally different human progesterone receptor forms A and B. EMBO fournal $\mathbf{9}$ $1603-1614$.

Mangal RK, Wiehle RD, Poindexter AN III \& Weigel NL 1997 Differential expression of uterine progesterone receptor forms A and B during the menstrual cycle. Foumal of Steroid Biochemistry and Molecular Biology 63 195-202.

McKenna NJ \& O'Malley BW 2002 Combinatorial control of gene expression by nuclear receptors and coregulators. Cell $\mathbf{1 0 8}$ $465-474$.

McKenna NJ, Lanz RB \& O’Malley BW 1999 Nuclear receptor coregulators: cellular and molecular biology. Endocrine Revieres 20 321-344.

Mesiano S, Chan EC, Fitter JT, Kwek K, Yeo G \& Smith R 2002 Progesterone withdrawal and estrogen activation in human parturition are coordinated by progesterone receptor A expression in the myometrium. Fournal of Clinical Endocrinology and Metabolism $872924-2930$.

Oberste-Berghaus C, Zanger K, Hashimoto K, Cohen RN, Hollenberg AN \& Wondisford FE 2000 Thyroid hormoneindependent interaction between the thyroid hormone receptor beta2 amino terminus and coactivators. Fournal of Biological Chemistry 275 1787-1792.

Onate SA, Boonyaratanakornkit V, Spencer TE, Tsai SY, Tsai MJ, Edwards DP \& O'Malley BW 1998 The steroid receptor coactivator-1 contains multiple receptor interacting and activation domains that cooperatively enhance the activation function 1 (AF1) and AF2 domains of steroid receptors. Foumal of Biological Chemistry 273 12101-12108.

Pieber D, Allport VC, Hills F, Johnson M \& Bennett PR 2001 Interactions between progesterone receptor isoforms in myometrial cells in human labour. Molecular Human Reproduction 7 875-879.

Sartorius CA, Melville MY, Hovland AR, Tung L, Takimoto GS \& Horwitz KB 1994 A third transactivation function (AF3) of human progesterone receptors located in the unique $\mathrm{N}$-terminal segment of the B-isoform. Molecular Endocrinology 8 $1347-1360$.

Tetel MJ, Giangrande PH, Leonhardt SA, McDonnell DP \& Edwards DP 1999 Hormone-dependent interaction between the amino- and carboxyl-terminal domains of progesterone receptor in vitro and in vivo. Molecular Endocrinology 13 910-924.

Tung L, Shen T, Abel MG, Powell RL, Takimoto GS, Sartorius CA \& Horwitz KB 2001 Mapping the unique activation function 3 in the progesterone B-receptor upstream segment. Two LXXLL motifs and a tryptophan residue are required for activity. Fournal of Biological Chemistry 276 39843-39851.

Vegeto E, Allan GF, Schrader WT, Tsai MJ, McDonnell DP \& O'Malley BW 1992 The mechanism of RU486 antagonism is dependent on the conformation of the carboxyl-terminal tail of the human progesterone receptor. Cell 69 703-713.

Vegeto E, Shahbaz MM, Wen DX, Goldman ME, O’Malley BW \& McDonnell DP 1993 Human progesterone receptor A form is a cell- and promoter-specific repressor of human progesterone receptor B function. Molecular Endocrinology 7 1244-1255.

Received in final form 1 December 2003 Accepted 31 December 2003

www.endocrinology.org 